\title{
Modelica Based Naval Architecture Library for Small Autonomous Boat Design
}

\author{
Thom Trentelman $^{1} \quad$ Joshua Sutherland $^{2} \quad$ Kazuya Oizumi $^{2}$ Kazuhiro Aoyama ${ }^{2}$ \\ ${ }^{1}$ Maritime Engineering, Delft University of Technology, The Netherlands, \\ t.r.trentelmanestudent.tudelft.nl \\ ${ }^{2}$ Systems Innovation, University of Tokyo, Japan, \{joshua, oizumi, aoyama\}@m.sys.t.u-tokyo.ac.jp
}

\begin{abstract}
This paper describes a method for early stage boat design by creating and utilizing a library of naval architecture based boat components in Modelica. The method involves the construction of stand-alone boat components which can be assembled into a simulation model. Structuring the model into multiple system levels provides a clear overview. Utilizing the partialcomplete methodology ensures that all system levels are replaceable within the simulation. This allows the user to construct many different boat models and experiment with unconventional or innovative designs. By comparing the performance and behaviour of different assemblies of components the most ideal design for a given purpose can be found and used as a starting point for the in-depth design process. By organizing the components in a library they can be re-used in future projects as well. It is noted that when additional libraries are utilized the effectiveness of this design method increases significantly. As the availability of component models increases, users can save time on the physical design and modelling of the individual components and instead focus on assembling working simulation models right from the beginning. To illustrate this, the construction of a few simple boat components is described in this paper. These components are then combined to simulate multiple concept designs.
\end{abstract}

Keywords: early stage ship design, model based design, object-orientated, innovative naval architecture

\section{Introduction}

Shipping nowadays is one of the world's most important means of transport. Not only for product or passenger transport, but also for leisure such as pleasure yachts and sport boats. The volume of world seaborne trade exceeded 10 billion tons in the year 2015. Together this volume was accountable for more than $80 \%$ of the total worldwide merchandise trade, growing with $2.1 \%$ over the year 2014 (UNCTAD/RMT, 2016). A downside to this key global trade enabler is the overall environmental pollution that comes from the industry. In 2012 ship emissions were responsible for $3.4 \%$ of the worldwide emissions of greenhouse gasses (GHG), ranking $2^{\text {nd }}$ as transport related polluter after road transport $(24.3 \%)$ and just above aviation $(3.1 \%)$. Although road transport is the major source of transport related emissions, shipping is the only sector where the GHG emission rates are still rising (Maragkogianni, Papaefthimiou, \& Zopounidis, 2016). A publication of the International Maritime Organisation (Buhaug et al., 2009) described a scenario where without political involvement the growth of ship's GHG emissions would grow by 150 250\% between 2007 and 2050.

\subsection{The Importance of Innovative Naval Architecture Design}

The design process of ships from scratch to seaborn is costly and requires large amounts of detailed analysis, is subject to extensive safety and environmental legislation and to remain competitive, the time-span for this design process is ever decreasing. There is little room for uncertainty or assumptions. And whilst most drastic and influential decisions are made in the early stages of the design process, most design software tends to emphasise more on the detailed phases in later stages of the design process (Abt, Bade, Birk, \& Harries, 2001; Bole \& Forrest, 2005). For these reasons ship design often loses its innovative character and mostly evolves around the slight improvement of existing designs.

However, due to international political involvement the imposed legislation on the emission of GHG has increased in the recent years. Stricter emission quotas and the introduction of eco-zones at major ports have forced the industry to innovate (Rue \& Anderson, 2015). Moreover, not only stricter legislation has moved the industry; in recent years the direct influence of global warming have exposed itself more clearly where conditions on sea have become more challenging for existing vessels due to the changing climate. Reports of an increase in storms and higher rising waves have set high performance demands for the vessels in order to maintain safety for their passengers and crew on board (Bitner-Gregersen, Eide, Hørte, \& Skjong, 2013). 


\subsection{A New Method for Innovative Early Stage Concept Design}

Using Model Based Design (MBD) in the early design stage makes it possible to experiment with innovative techniques. For example, 3-Dimensional simulation gives direct performance output of the created concept. However, the level of detail of the simulation output will vary greatly based on the complexity of the simulation model. This results in the necessity for further investigation. This in-depth design stage follows a spiral path. Such a design path has an iterative character; the concept design should run down all design stages multiple times. At the end of every iteration the concept design should be adjusted to ultimately result in a suitable design (Papanikolaou, 2014). Even though eventually this high detail design path is inevitable for every concept design, this method only states whether the chosen concept is suitable for its goal or not after one or more iterations. When the concept turns out to be unsuitable for its goal, the process must start over from the very beginning. Although this method allows innovative decisions to made regarding concept designs, it does not solve the time issue.

In this paper, we present a complimentary method utilizing the Modelica modelling language in the early design stages, with the simulation model subdivided into multiple system levels. The advantage is that these system levels have a replaceable properties, with every system level consisting of one or more components. The components can be anything the user wants to attach to his simulation model e.g. thrusters, motors, solar panels, gearboxes and batteries. Connecting these components all the way through to the top level creates a complete simulation model arranged in a hierarchy to aid the management of complexity. By swapping components within these levels multiple concepts are established on the same structure. Still the level of detail of the simulation output is heavily reliant on the complexity of these individual components. However, at this design phase the suitability of the designs can be determined by comparing the performance of various concepts. Only the most promising concept designs will be subjected to the advanced design stage. Meanwhile all created components can be exchanged or stored in a library for use in later projects.

\section{Background to Model Based Design and its Application in Navel Architecture}

\subsection{Model Based Design}

Model Based Design (MBD) is a design method which enables the rapid creation of design concepts in software by leveraging advances in computing technology.

While various modelling languages exist their choice to be used on a specific project and lifecycle stage within that project must be made based on what value they provide. Conceptual modelling languages such as SysML provide a structure to describe a system and its purpose, but fail to provide feedback on the performance of the system being designed. Conversely 3D CAD, can give clear and direct feedback on the geometry of a system but requires the designer to commit to a great deal of detail which often occurs later in the design lifecycle.

1 Dimensional Computer Aided Engineering (1D CAE) is a broad term used to cover methodologies and tools which aid the early stages of engineering lifecycles by the utilization of computers (Sawada, 2012). By deliberately neglecting 3D geometry the engineer can quickly prototype designs and crucially; predict the performance by simulating the models created rather than creating a physical prototype. Likely leading to cost and time savings for a given project and making it easier to complete difficult trade-off decisions which must made to select a combination of components to form a system which serves its purpose best.

Modelica is an example of a numerical modelling language popular in the 1D CAE paradigm. Equations capture the behaviour of individual components which are then connected together to develop subsystems which ultimately form the system being modelled. Its object orientated features make it possible to quickly create and modify models including the sharing of interfaces and inheritance of common attributes. Features of which are vital for the handling highly complex systems being developed by large teams.

This is particularly useful when attempting to create highly innovative designs where experimental or extraordinary components can be realized as models by their particular domain experts and integrated into a system by an engineer who is not a specialist in those particular domains.

\subsection{Current Applications of Modelica to Naval Architecture}

A review of the current literature shows the use of Modelica in the naval architecture has been focusing on the design of highly detailed specific systems aboard ships. For example (Dong, Wu, Zhang, \& Peng, 2011) have constructed a simulation model of a hydraulic rudder and used it for the analysis of shock resistant effects while (Marty, Corrignan, Gondet, Chenouard, \& Hétet, 2012) focussed on simulating energy flows and fuel consumption on board of a large cruise vessel.

The high level of detail of these simulation models is typical for the advanced design stage of a ship or boat and as such these stand-alone simulations are not meant to be integrated into a larger holistic simulation model of the entire ship or boat. The amount of detail in these individual component models would be excessive for the construction of an early design stage model. 
Through the course of a literature review no prior attempt at using Modelica to model an entire ship or boat was found. As such we conclude the navel architecture preliminary design process is likely still reliant on other software languages other than Modelica and so is not experiencing the benefits described in Section 2.1 of this paper.

\section{Contributions of this Paper}

Earlier literature from authors including (Sutherland, Oizumi, Aoyama, Eguchi, \& Takahashi, 2016; Sutherland, Oizumi, Aoyama, Takahashi, \& Eguchi, 2016; Sutherland, Salado, Oizumi, \& Aoyama, 2017) has focused on the practical use of a Modelica based naval architecture library. It describes the process of designing a race winning model boat for an annual student contest. The boat model needed to be rather innovative as it was assigned to sail autonomously on solar energy. This paper will describe the details and architecture of the navel architecture library used.

The focus will be on the replaceable components, system level characteristics and the hierarchal structure. Successively the architecture of several components will be described to aid reader comprehension.

Through this paper the authors aim to inspire engineers from the naval community to take benefit from advanced design methods and tools. The effectiveness of the design method will grow exponentially as more naval component model libraries become available.

\section{Methodology for Creating Replaceable System Levels}

As shown in Figure 1 the simulation model is subdivided into four systems levels. All system levels are linked to one another and ultimately make up the Solar Boat Model. All system levels are replaceable since they are all constructed using the partial-complete methodology. With one click a component, subsystem or system of interest can be swapped for an alternative.

\subsection{System Levels}

The hierarchical structure provided by the four system levels offers a clear overview of the simulation model as demonstrated in Figure 1. In Modelica these system levels are introduced as separate packages. Underneath these, every system level has their own sub-packages containing relevant models.

At the bottom level (Level 4) all individual components are arranged in a library. This library provides fundamental elements to the simulation model, where other system levels are introduced for enabling quick and drastic concept changes. The number of possible components is infinite and the level of complexity per component is variable. Components can often be broken down into separate sub-components. These sub-components can then be reconnected at the subsystem level. However, it is important to acknowledge that this method is used in the early (lower detail) design stage.

The subsystem level (Level 3) contains assemblies of Level 4 components which interact one-on-one to function properly in a system. Every unique
Level 1

Simulation

level

Level 2

Systems of Interest

Level 3

Subsystems

Level 4

Component library
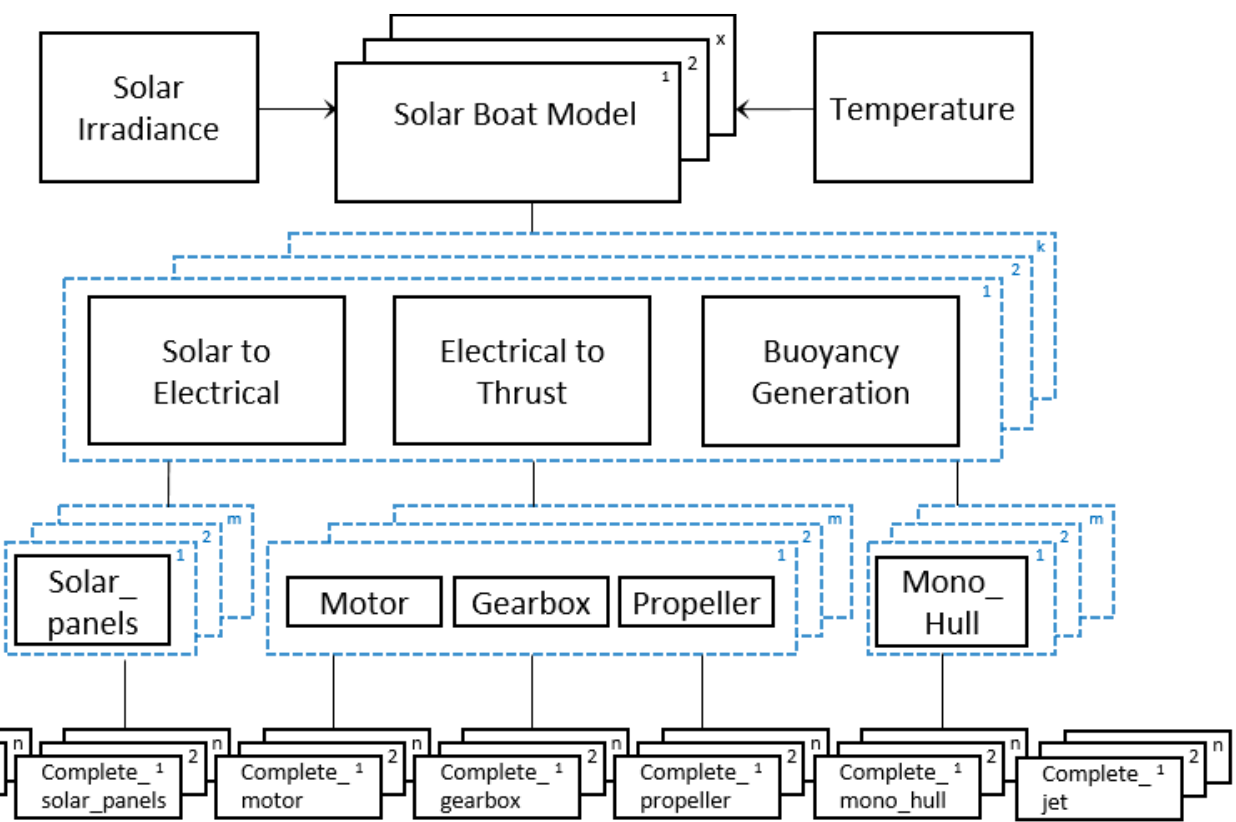

Figure 1. Schematic view of system level hierarchy; enabling the creation of several ready to simulate Solar Boat models by alternating various replaceable complete components from the library (Level 4). System Levels 2 and 3 are also replaceable as shown in Figure 4 and Figure 5. 
combination of components within a subsystem requires a different subsystem name at this level.

The systems of interest level (Level 2) combines all subsystems which will be used in the concept. It is at this level that changes will radically influence the appearance of the concept design.

At the top level (Level 1) the final concept design is assigned and connected with external factors. These external factors could be weather conditions, but also payloads or obstacles in the simulated space the vehicle operates in. At this level the model is complete and ready to simulate.

\subsection{Partial-Complete Methodology}

The partial-complete methodology, as the name suggests, makes use of a partial model to create multiple complete models of the same type. This objectedorientated inheritance scheme uses a partial model to state the characteristic parameters and equations for every component, subsystem or system of interest. Each complete model then extends their corresponding partial model and assigns real values to the parameters. The partial-complete methodology allows the models to be replaceable and enables the rapid creation of multiple complete components using the same architecture. Figure 2 shows a flow-chart which demonstrates the methodology for a simplified (Level 4) component.

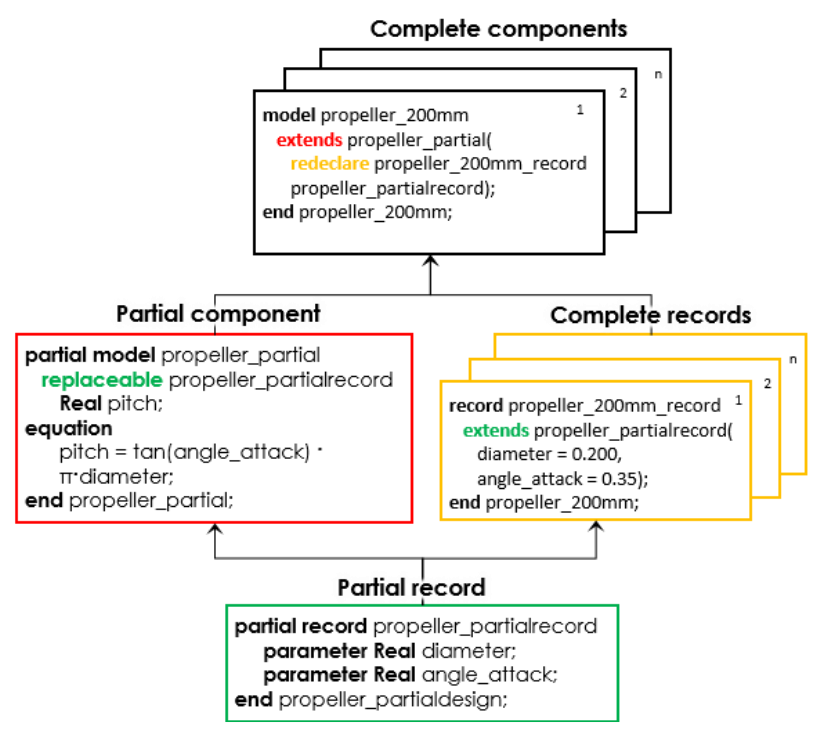

Figure 2. Flow chart demonstrating the partial-complete methodology for creating a replaceable propeller component (Level 4). The component parameters and equations have been heavily simplified.

\subsubsection{Component Modelling (Level 4)}

Not only do all components have to be replaceable, for experimenting it is also desirable to make the components easily adjustable and scalable. The use of records ensures this. A record formulates all constant parameters which are used in the physical description of the component. The very base of a component is a partial record.
A partial record states all the parameters without assigning a value to them. For example, these parameters could be dimensions, mass, cost, conductivity, material characteristics and cost. A complete record extends the partial record and ultimately assigns values to the parameters. By creating various complete records with alternating values, every time extending the same partial record, many different components of the same type can be created at the one instant. Using the partial-complete methodology on a record level ensures that components of the same type are using identical parameters.

The partial component is used to describe the outlines of the component and can include both interfaces and behaviour. When used for describing the outline of a component, it must declare replaceable the partial record and therefore gain access to the parameters. These parameters must then be complemented with additional variables which will solely be used in the characteristic set of equations of the component. Note that every time a component desires a new set of equations, a new partial component has to be created.

In order to make the components connectable to other components all output forces expressed by the different components must be attached to the same frame. To enable this a connector from the Modelica Mechanics.Multibody library is used in the Diagram view of the partial specifically: Interfaces.Frame a. By using this library the Modelica compiler can automatically combine the forces and torques excreted by the components and compute the resulting net acceleration on the entire boat assembly. In some cases (e.g. components affected by hydrodynamics) it is useful to attach Mechanics.Multibody. Sensors to the frame. The data from this sensors could be used in the equations of the same partial model (e.g. compute drag from velocity). Lastly, for a clearer understanding of the 3D simulation of the model Mechanics.Multibody. Visualizers can also be attached to the frame. The template for the Diagram view is shown in Figure 3.

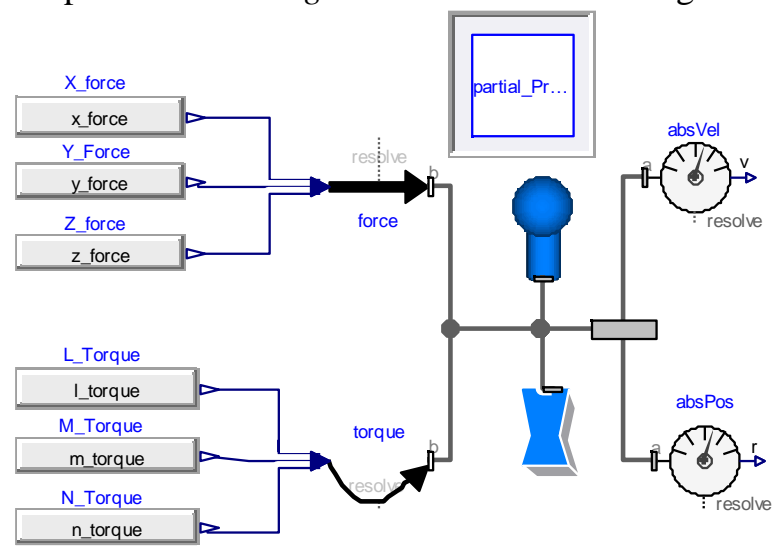

Figure 3. Diagram template used for creating general boat components (Level 4). 
The last step for creating a Level 4 component is to create a complete component. The complete model extends its corresponding partial component and inherits the values for the parameters by re-declaring the partial record with the chosen complete record. Every complete record should be re-declared in a complete model in order to create the eventual replaceable components.

\subsubsection{Subsystem Modelling (Level 3)}

Subsystems (Level 3) are models that state one or more component types which the designer decides should be grouped together. In the partial subsystem the partial components are assigned. Constraining the replaceable partial components to their own types (complete components) makes a subsytems distinctive. This is illistrated in the following Modelica code for a partial Electrical to Thrust partial subsystem which is also shown as a diagram in the central layer of Figure 4:
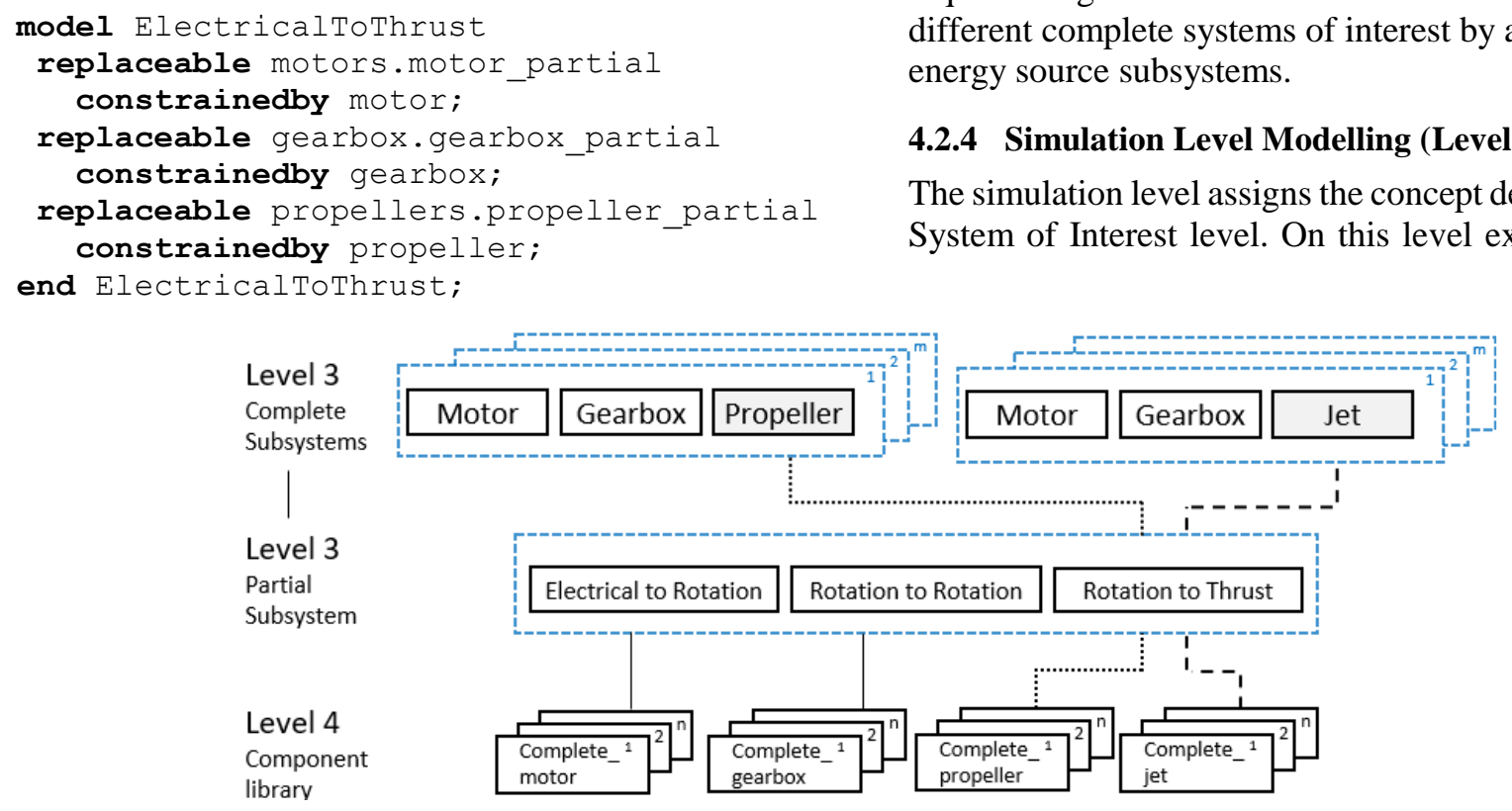

Figure 4. Schematic view of the partial-complete methodology as used for the creation of replaceable subsystems (Level 3 ). In this specific example both replaceable complete subsystems will use a motor and a gearbox. The difference comes from the variation of propeller (dotted line) and jet (dashed line) as main thruster.

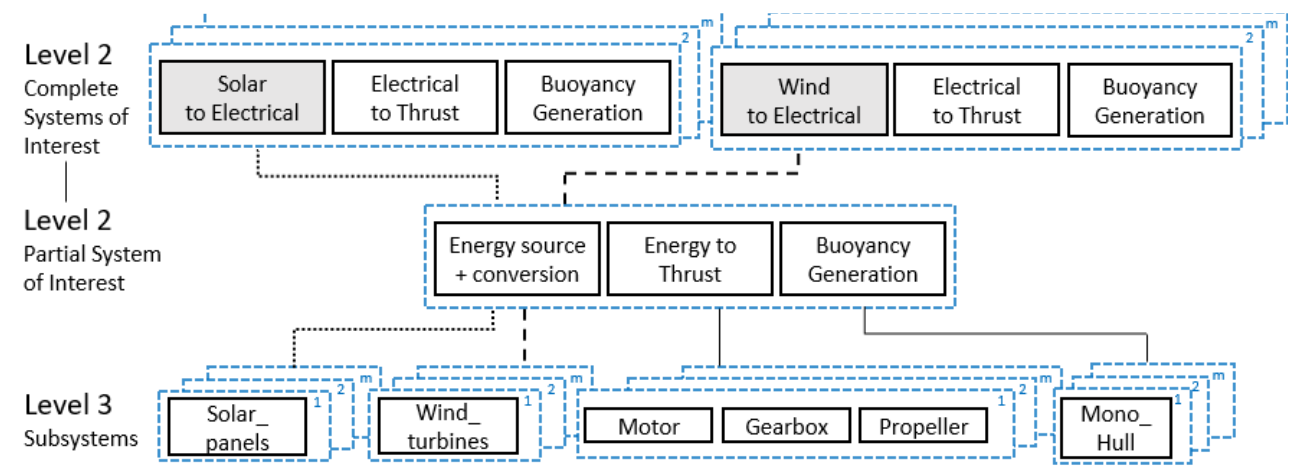

Figure 5. Schematic view of the partial-complete methodology used for the creation of replaceable systems of interest (Level 2). In this specific example both replaceable complete systems of interest will use a motor, gearbox and propeller propulsion system, as well as a mono-hull buoyancy generation. The difference comes from the variation of solar panels (dotted line) and windmills (dashed line) as main energy source. 
such as weather can be linked to the concept design, making it ready to simulate.

\section{The Modelica Naval Architecture Library}

This chapter demonstrates the practical application of the design method as described in Chapter 4 in order to construct a complete simulation model. The simulation model will be a solar powered model boat. Although this design method has the potential to model the simulation model with six degrees of freedom (see Figure 3), for reasons of simplification this paper will model in only two degrees of freedom. This means that the eventual simulation output will only contain a boat travelling in a straight line and moving up and down along its vertical axis.

\subsection{The Library: Component Modelling (Level 4)}

This section describes the individual components which are used in the simulation model. Even without precise knowledge about the physics of every type of component, it is still possible to construct functioning components by yourself. This method allows using existing literature to formulate component characteristics and equations. This makes it possible to construct components even without knowledge or thorough research. In this paper the solar panel components are based on existing literature. In some occasions components, such as motors and gearboxes, could be implemented from the Modelica Standard Library. As a result, very few parameters and equations have to be formulated in the partial models, keeping the components simple. In other occasions the components will be based on the authors' expertise, resulting in more complex, yet more adjustable components (as shown with the propeller and buoyancy components later in the paper).

\subsubsection{Solar Panel Components}

The solar panel components are implemented as modelled by (Esram, 2010). Most parameters are predefined and do not need to be changed. However, for simulation purposes the solar panel components needs additional parameters such as surface area, mass and cost. These additional parameters should be formulated in the partial record along with the other pre-defined parameters and later assigned by a complete record in a complete component.

Also it is possible to vary the distribution of the solar panels over the length of the boat model. Every unique distribution requiring a different partial component, later extended by corresponding complete components.

For a solar panel to generate electricity it needs solar irradiation. This solar radiation needs to be modelled on the simulation level (Level 1). In order for this external value to reach the solar panel component it needs to be implemented as an input in the Modelica Diagram view of the partial solar panel component.

\subsubsection{Electrical Motor and Gearbox Components}

The electrical motor component and the gearbox component are selected from the Modelica Standard Library. Without any adjustment these component will respond to the appropriate inputs and generate an output. These inputs and outputs need to be connected within a subsystem, and on the system of interest level.

Both the electrical motor and the gearbox need some additional adjustment before they can be successfully implemented in the simulation model. Just like the solar panel component, the motor and gearbox need to have parameters for size, mass and cost assigned. Also, it is important to determine a gearbox ratio. Which of course is also adjustable.

Every time an adjustment in one of the parameter values is desired, a new complete component has to be created, extending the partial component.

\subsubsection{Propeller Component}

The propeller is a propulsion device often mounted at the stern of the boat. Although there are many different types of propulsion devices, the propeller is the most commonly used. A boat can be designed having more than one propeller. The direction of the propeller can be fixed or with the ability to rotate around its vertical axis in order to adjust the direction of the boat, like the propellers on typical outboard motors. In this simulation, a fixed single propeller is used as the thruster of the boat.

The propeller generates the thrust which will ultimately displace the boat in the simulation. The thrust is calculated using the basic equation:

$$
\text { Thrust }=k_{t} \cdot \rho \cdot n^{2} \cdot D^{4}
$$

At the same time a propeller generates a torque. This toque is described by using a similar basic equation:

$$
\text { Torque }=k_{q} \cdot \rho \cdot n^{2} \cdot D^{5}
$$

Where $(\rho)$ is the density of the water, $n$ is the revolutions per second of propeller, $D$ the diameter of the propeller and $\left(k_{t}(J)\right)$ and $\left(k_{q}(J)\right)$ are the parametrised thrust and torque coefficients, respectively. Since $\rho$ is determined by the environment and $n$ is as a result of interaction with torque from either the gearbox or the motor, only the diameter and the coefficients have to be formulated in the partial record of the propeller.

The coefficients are found by a $4^{\text {th }}$-order polynomial in the form of $a \cdot J+b \cdot J^{2}+c \cdot J^{3}+d \cdot J^{4}$, where $J$ is the advance ratio of the propeller. The advance propeller is determined using the equation: 


$$
J=\frac{V_{a}}{n \cdot D}
$$

Where $V_{a}$ stands for the advance velocity (the velocity of the water as it reaches the propeller, which in practice is influenced by the shape of the hull). For simplification, the advance velocity in this simulation is equalled to the absolute velocity of the simulation, the value for which is extracted from the implemented velocity sensor.

The $a, b, c$ and $d$ polyfits within the $4^{\text {th }}$ order polynomial are determined for a particular propeller design by iterative calculation using a calculation executed in Matlab as described by the blade element theory presented by (Auld \& Srinivas, 2016). But of course experimentally derived functions could also be used.

\subsubsection{Buoyancy Component}

The buoyancy component describes the hull used in the simulation and the interaction with its surrounding. The hull of a boat can come in various shapes and sizes. In addition there is possibility to design a mono-hull, double-hull or even a triple-hull. The shape and weight of the hull mainly affects the resistance from the water and the stability of the boat. However its hydrodynamic outline and the interaction with the water are extremely difficult to accurately model without resorting to calculations within the field of advanced 3D fluid dynamics. But in the preliminary design stage it is not necessary to calculate so accurately and some basic equations are sufficient as described in the following subsections.

\subsubsection{Resistance force}

To reduce complexity the resistance of the buoyancy component calculation is primarily calculated by the friction force (Equation 4) using the ITTC-57 equation (Equation 5).

$$
\begin{gathered}
R_{\text {total }}=R_{\text {friction }} \\
R_{\text {friction }}=\frac{1}{2} C_{f} \cdot \rho \cdot S \cdot V^{2}
\end{gathered}
$$

Where $C_{f}$ is the friction coefficient, $\rho$ is the density of the water, $S$ is the wetted surface area of the hull and $V$ is the absolute velocity of the model.

The friction coefficient itself is a function of the number of Reynolds Number, hence the following equations:

$$
C_{f}=\frac{0.075}{\left(\log _{10}(R e)-2\right)^{2}}
$$

$$
R e=\frac{V \cdot L_{w l}}{v}
$$

Reynolds Number as shown in equation 7 is calculated using the length of the waterline $\left(L_{w l}\right)$ of the hull rather than the overall length but for simplification this could be equalled to the overall length. It is multiplied with the absolute the velocity and then divided by the kinematic viscosity of the water $(v)$. This parameter is determined as a function of temperature and water density, but could also be implemented as a constant. For salt water with a density of $1025 \mathrm{~kg} / \mathrm{m}^{3}$ and a temperature of $15^{\circ} \mathrm{C}, v=1.188 \cdot 10^{-6}$ is used.

The wetted surface $(S)$ is for simplification equalled to the surface of the under half of an ellipsoid and assumed to be constant:

$$
S=\frac{1}{2} \cdot 4 \pi \frac{\left((a b)^{1.6}+(b c)^{1.6}+(a c)^{1.6}\right)^{\frac{1}{1.6}}}{3}
$$

Where $a$ is the overall length of the hull, $b$ the greatest width and $c$ the depth, vertically measured from the bottom to top of the ellipsoid.

The frictional resistance always works in the opposite direction of the traveling direction of the boat. More resistance components such as air resistance and wavemaking resistance could be added in order to increase the accuracy of the resistance simulation but they are neglected at this time.

\subsubsection{Buoyancy force}

The buoyancy force keeps the vessel floating on the water and maintains its stability. It has an upward force component along the $\mathrm{z}$-axis and restoring moments around the $\mathrm{x}-, \mathrm{y}-$ and $\mathrm{z}$-axes of the construction. In the simulation the restoring moments have been neglected for simplification. The equation for the buoyancy force along the $\mathrm{z}$-axis then becomes:

$$
F_{\text {buoyancy }}=\nabla \cdot \rho \cdot g
$$

The water displacement $(\nabla)$ would normally be calculated using the block coefficient, length of the water line, breadth and draft of the hull. The equation is as follows:

$$
\nabla=C_{b} \cdot L_{w l} \cdot B \cdot T
$$

However, to remain consistent with previous calculations, the displacement will again be calculated using an ellipsoid equation:

$$
\nabla=\frac{1}{2} \cdot \frac{3}{4} \pi a b c
$$


Again only the bottom half of the ellipsoid will be submersed. The coefficients $a b c$ are used the same as in equation 8 .

\subsubsection{Overhead Components}

The overhead components make up for weight or cost values of components which have not been modelled or used in the simulation model. Moreover they could be used as the safety margin of the design. The overhead components have no further dimensions or forces applied other than weight.

\subsection{The Library: Subsystems (Level 3)}

The subsystems which are used in the boat model are Solar to Electrical, Electrical to Thrust, Buoyancy Generation and Overhead Structures (as shown in Figure 1, overhead structures excluded).

\subsubsection{Solar to Electrical}

The Solar to Electrical subsystem consists of solely the solar panels. The distribution of solar panels is determined at this level. The output from the solar panels is an electrical connection which has to be connected to an electrical motor. In the subsystem an Electrical. Analog. Interfaces. PositivePin and a .NegativePin are utilized. In the systems of interest level these pins can be used to connect the electrical current to the electrical to thrust subsystem (see Figure 9). Figure 6 show a possible composition for the Diagram view of the Solar to Electrical subsystem.

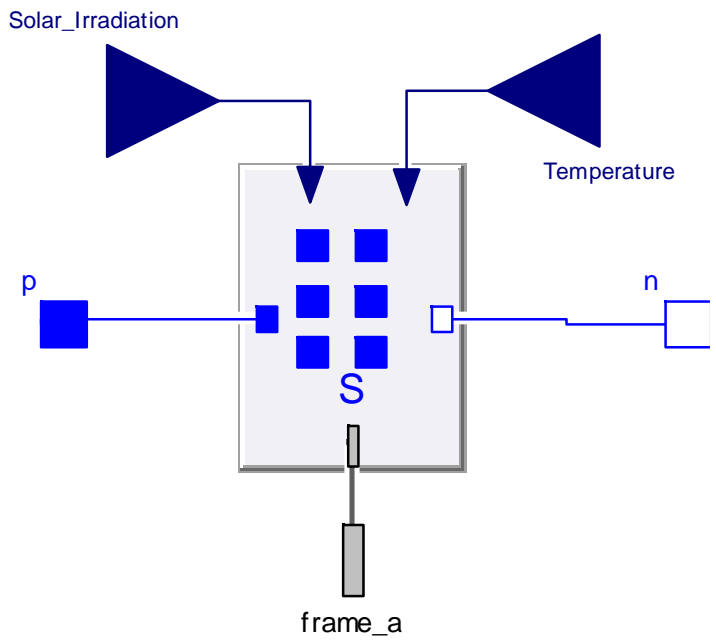

Figure 6. Diagram view of Solar to Electrical subsystem.

\subsubsection{Electrical to Thrust}

The electrical to thrust subsystem is responsible for the transformation of the electrical current generated by the solar panels into a thrusting force. It accesses electrical current via the connection of the pins at the system of interest level (Level 2).

Several configurations could be made to convert the electrical signal and the resulting thrusting force with every configuration should be constructed in a separate partial subsystem.
Figure 7 shows a Diagram view of an electrical to thrust subsystem containing a motor, gearbox and propeller. These components are mechanically linked to each other. The motor component has two wires with pins attached, which will be used to link this components in the system of interest level to the energy source (see Figure 9).

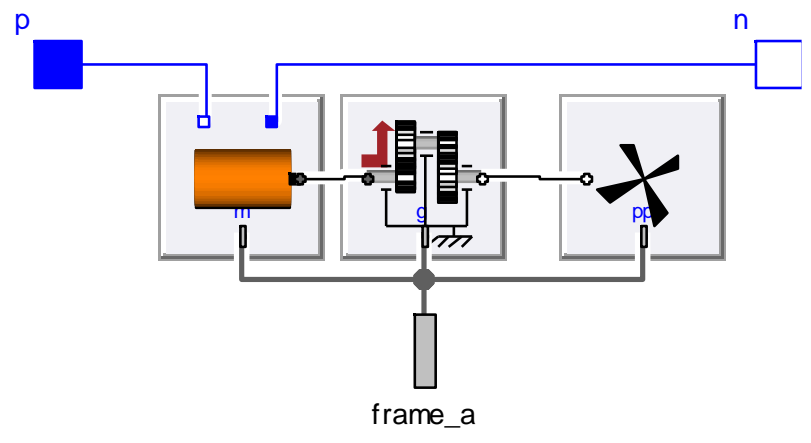

Figure 7. Diagram view of Electrical to Thrust subsystem.

\subsubsection{Buoyancy Generation}

The buoyancy generation subsystem is used to connect the hull component with external factors such as water velocity or wind speed. A "single hull" subsystem will contain only one hull component.

If the concept design should contain multiple hull components, as seen on catamaran or trimaran boats, a separate partial subsystem must be created. Two or more replaceable components can be implemented and attached to the frame.

Figure 8 show a Buoyancy Generation subsystem with a single hull component, connected to a water velocity input.

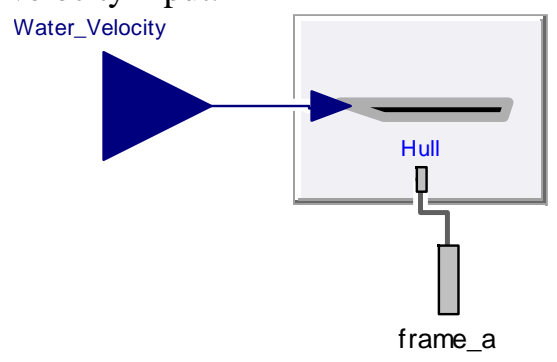

Figure 8. Diagram view of Buoyancy Generation subsystem.

\subsubsection{Overhead Structures}

The overhead structures subsystem is a stand-alone subsystem which assesses the overhead structure component of choice and make it possible to fit this into the simulation in the systems of interest level.

\subsection{The Library: Systems of Interest (Level 2)}

The systems of interest model combines various subsystems to form a model of the system for assessment (i.e. a boat). At this system-level (Level 2) it is possible to change entire subsystems with the use of the partial-complete methodology described previously. 


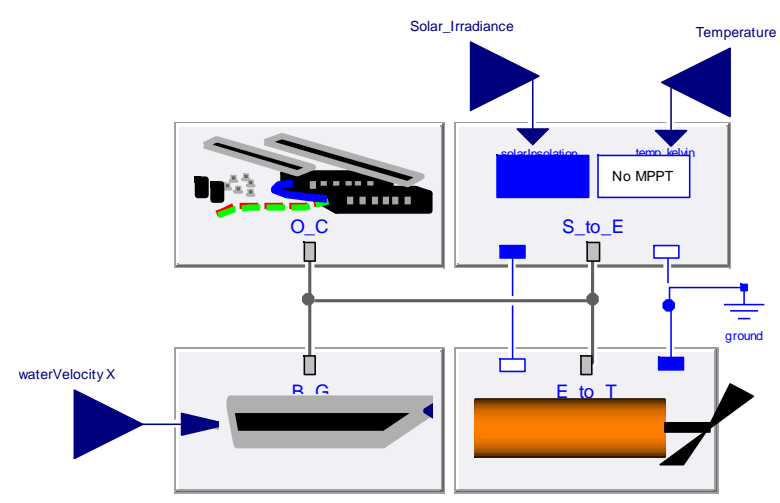

Figure 9. Diagram view of Systems of Interest (Level 2).

\subsection{The Library: Simulation Level (Level 1)}

The simulation level contains the chosen concept design and connects it to all the external parameters such as environmental elements or payloads. It is at this level that the simulation is ready to run. Chapter 6 gives an example of simulating multiple concept designs.

\section{Simulation}

In this section we present the results of simulating boat concept designs constructed in Modelica. With the use of 3D animation (by means of Mechanics. Multibody library) it is possible to visualize the behaviour of a particular concept (see Figure 10). While detailed performance can be analysed using charts. When multiple different concept designs are simulated, the data from the charts can be compared to determine the most suitable design for its purpose.

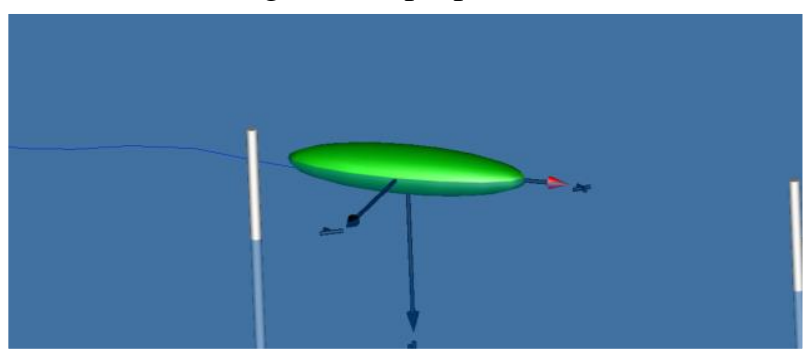

Figure 10. Screenshot of the 3-Dimensional simulation.

\subsection{Simulating the Concepts}

To demonstrate, we compare the results of simulating two competing boat designs. Both designs are based on the basic set of components as described in Chapter 5 . However, they vary with Concept 1 being powered by mid-range efficiency solar panels $(12.5 \%, 3.24 \mathrm{~kg})$. Demanding a higher maximum boat velocity, the second simulation will be powered by more efficient, however slightly heavier solar panels $(21.5 \%, 5.40 \mathrm{~kg})$. It should be noted that the maximum allowable solar panel surface may not exceed $2 \mathrm{~m}^{2}$. Six mid-range efficiency solar panels almost perfectly fill up all the available space, where six of the high efficiency solar panels only fill up $89 \%$ of all the available space. There is no room left for adding a seventh solar panel. By simulating both boats subject to the same environmental conditions of average solar irradiance of a day in August in Japan (610 $\mathrm{W} / \mathrm{m}^{2}$ ) it is possible to construct Figure 11 with time series of velocity and Table 2 containing data selected from the point when the simulation reached a steady state (simulation time $=25 \mathrm{~s}$ ). As mentioned previously no steering component is attached to the boat and hence the boat only moves in the $\mathrm{X}$ and $\mathrm{Z}$ axis.

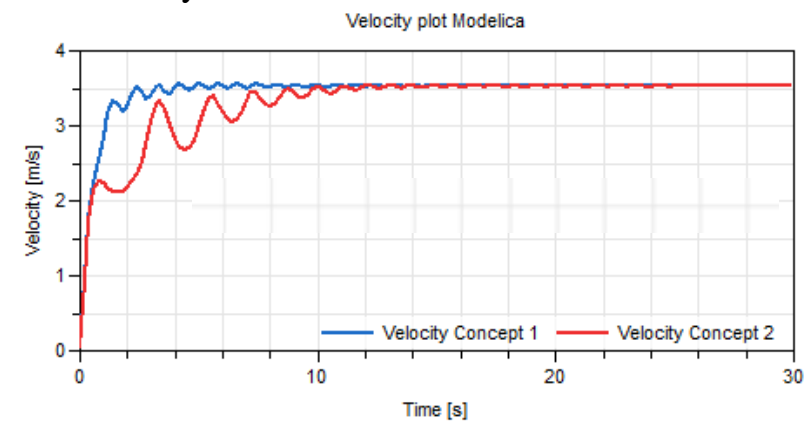

Figure 11. Simulation results of two concept designs.

Table 2. Simulation data of two concept designs.

\begin{tabular}{|l|c|c|}
\hline Concept & $\mathbf{1}$ & $\mathbf{2}$ \\
\hline Efficiency of solar panels [\%] & 12.5 & 21.5 \\
\hline Area per solar panel $\left[\mathrm{m}^{2}\right]$ & 0.333 & 0.297 \\
\hline Area of six solar panels $\left[\mathrm{m}^{2}\right]$ & 1.99 & 1.78 \\
\hline Mass of six solar panels [ kg] & 3.24 & 5.40 \\
\hline Spin speed motor [rpm] & 12900 & 13300 \\
\hline Thrust generated [N] & 111 & 118 \\
\hline Draft boat [m] & 0.166 & 0.185 \\
\hline Maximum velocity [m/s] & 3.56 & 3.53 \\
\hline
\end{tabular}

\subsection{Analysing the Simulation Results}

The expectation would be that the design with the most efficient solar panels, generating more power, will result in a faster concept. However, by looking at the output results from both simulations we find both concepts reaching about the same maximum velocity. We therefore conclude that the more efficient solar panels, used in Concept 2, are not appropriate to increase the velocity of the boat.

In order to increase the velocity of the boat we could run more simulations with different hull designs in order to decrease the draft and ultimately the drag, swap motors, gearboxes and propellers or simply try to find lighter solar panels with a sufficient efficiency.

\section{Discussion}

\subsection{Benefits}

By using the design strategy utilizing Modelica described in this paper for preliminary ship design engineers are able to rapidly construct working assemblies and cut valuable time on their early design research. Even without detailed domain knowledge of naval architecture a user is able to assemble and compare different models. 
By using Modelica's object oriented features the methodology enables radical or minor changes to a simulation model with one mouse-click. With the possibility to directly run a $3 \mathrm{D}$ animation of the simulation model the reaction to these changes can be reviewed immediately.

There is basically no limit of components to add to the simulation and it is also it is possible to break up existing components into several separated components. For example, a rudder can be simply modelled by the deflection of alternating a single value for the angle, or if desired be split up into different components, where the mechanism to initiate this deflection is implemented as individual components.

\subsection{Limitations}

As for every design method, the simulation output will never be an exact representation of reality. The methods used for describing the boat components are by necessity based on approximations. Given the stated goals of the 1DCAE method can only be used for preliminary ship design, the engineer must still run detailed design iterations on the eventual chosen model in order to create a system which is producible and successful.

\section{Conclusions and Future Work}

\subsection{Conclusion}

This paper set out to describe a new method for the early stage design of ships which are highly innovative. We achieved this by introducing the use of Modelica for complete ship design. Combined, they form a working simulation of a boat that travels in a straight line. While the accuracy of the simulation may not be very high, at this stage it is already possible to determine the difference between the performance and behaviour of different assemblies. Only once the demands for the eventual design rise, must the simulation increase in accuracy.

\subsection{Future Work}

Primarily the content of the library must be expanded in the future. Further, the availability of many different types of components will make it possible to assemble innovative simulation models. Therefore, the authors hope to see more marine engineers finding their way to Modelica.

In addition, given the complexity level can vary per component and every ship design has its own purpose and requires different accuracies from the simulation a balance must be made on how complex to make each component. However, if for example waves are to be simulated all forces in the six degrees of freedom must be formulated in every component. Which in turn will make the library more useful.

\section{References}

Abt, C., Bade, S., Birk, L., \& Harries, S. (2001). Parametric hull form design-a step towards one week ship design. In 8th international symposium on practical design of ships and other floating structures (pp. 67-74).

Auld, \& Srinivas. (2016). Blade Element Propeller Theory. Retrieved November 10, 2016, from http://s6.aeromech.usyd.edu.au/aerodynamics/index.php/sa mple-page/propulsion/blade-element-propeller-theory/

Bitner-Gregersen, E. M., Eide, L. I., Hørte, T., \& Skjong, R. (2013). Ship and offshore structure design in climate change perspective. Springer.

Bole, M., \& Forrest, C. (2005). Early stage integrated parametric ship design. In Proc. ICCAS (pp. 447-460).

Buhaug, Ø., Corbett, J. J., Endresen, Ø., Eyring, V., Faber, J., Hanayama, S., ... others. (2009). Second IMO GHG Study 2009. London UK: International Maritime Organization.

Dong, R., Wu, C., Zhang, J., \& Peng, W. (2011). Modeling and simulation for ship hydraulic rudder system based on Modelica/MWorks [J]. Ship Science and Technology, 11, 20.

Esram, T. (2010). Modeling and Control of an AlternatingCurrent Photovoltaic Module. University of Illinois at Urbana-Champaign.

Maragkogianni, A., Papaefthimiou, S., \& Zopounidis, C. (2016). Shipping Industry and Induced Air Pollution. In Mitigating Shipping Emissions in European Ports (pp. 19). Springer International Publishing.

Marty, P., Corrignan, P., Gondet, A., Chenouard, R., \& Hétet, J.-F. (2012). Modelling of energy flows and fuel consumption on board ships: application to a large modern cruise vessel and comparison with sea monitoring data. In Proceedings of the 11th International Marine Design Conference, Glasgow, UK (pp. 11-14).

Papanikolaou, A. (2014). Ship Design: Methodologies of Preliminary Design. Athens Greece: Springer.

Rue, C. D. L., \& Anderson, C. B. (2015). Shipping and the Environment. New York USA: Routledge.

Sawada, H. (2012). Upstream design and 1D-CAE. Journal of System Design and Dynamics, 6(3), 351-358.

Sutherland, J., Oizumi, K., Aoyama, K., Eguchi, T., \& Takahashi, N. (2016). System-Level Design Tools Utilizing OPM and Modelica. In ASME 2016 International Design Engineering Technical Conferences and Computers and Information in Engineering Conference IDETC2016. Charlotte, North Carolina.

Sutherland, J., Oizumi, K., Aoyama, K., Takahashi, N., \& Eguchi, T. (2016). System-Level Design Trade Studies by Multi Objective Decision Analysis (MODA) utilizing Modelica. In The First Japanese Modelica Conference, May 23-24, Tokyo, Japan (pp. 61-69). Tokyo Japan: Linköping University Electronic Press.

Sutherland, J., Salado, A., Oizumi, K., \& Aoyama, K. (2017). Implementing Value-Driven Design in Modelica for a racing solar boat. In 15th Annual Conference on Systems Engineering Research. Los Angeles, California (accepted for presentation).

UNCTAD/RMT. (2016). Review of Maritime Transport 2016. Geneva Switserland: United Nations Publication. 